\title{
Identification of emerging viral genomes in transcriptomic datasets of alfalfa (Medicago sativa L.)
}

\author{
Peng Jiang, Jonathan Shao and Lev G. Nemchinov* (i)
}

\begin{abstract}
Background: Publicly available transcriptomic datasets have become a valuable tool for the discovery of new pathogens, particularly viruses. In this study, several coding-complete viral genomes previously not found or experimentally confirmed in alfalfa were identified in the plant datasets retrieved from the NCBI Sequence Read Archive.

Methods: Publicly available Medicago spp. transcriptomic datasets were retrieved from the NCBI SRA database. The raw reads were first mapped to the reference genomes of Medicago sativa and Medigago truncatula followed by the alignment of the unmapped reads to the NCBI viral genome database and de novo assembly using the SPAdes tool. When possible, assemblies were experimentally confirmed using 5'/3' RACE and RT-PCRs.

Results: Twenty three different viruses were identified in the analyzed datasets, of which several represented emerging viruses not reported in alfalfa prior to this study. Among them were two strains of cnidium vein yellowing virus, lychnis mottle virus and Cactus virus $X$, for which coding-complete genomic sequences were obtained by a de novo assembly.

Conclusions: The results improve our knowledge of the diversity and host range of viruses infecting alfalfa, provide essential tools for their diagnostics and characterization and demonstrate the utility of transcriptomic datasets for the discovery of new pathogens.
\end{abstract}

Keywords: Alfalfa (Medicago sativa L.), Transcriptomic discovery, Plant viruses

\section{Background}

Alfalfa (Medicago sativa L.) is one of the most extensively cultivated forage legumes in the world [1]. It has recently become the third most valuable field crop in the United States with an estimated worth of over $\$ 9.3$ billion, which is $\$ 1.2$ billion more than that of wheat, according to the National Alfalfa and Forage Alliance [2]. Alfalfa productivity is often limited by various biotic and abiotic components in the ecosystem $[1,3,4]$. With the increase in the production of monocropped alfalfa, infectious diseases, including viruses, have became more common. Traditionally, viral infections of alfalfa have been considered by producers, breeders, growers and research communities as diseases of limited importance.

\footnotetext{
* Correspondence: lev.nemchinov@usda.gov

USDA/ARS, Beltsville Agricultural Research Center, Molecular Plant Pathology Laboratory, Beltsville, MD 20705, USA
}

(c) The Author(s). 2019 Open Access This article is distributed under the terms of the Creative Commons Attribution 4.0 International License (http://creativecommons.org/licenses/by/4.0/), which permits unrestricted use, distribution, and

Nonetheless, they are widespread in major alfalfa cultivation areas and their contribution to the severity of complex infections involving multiple pathogens is poorly understood. Recently, many emerging viral diseases of alfalfa have been described that have the potential to cause serious yield losses. These include a rhabdovirus that was diagnosed in alfalfa plants displaying multiple abnormalities [5]; a new enamovirus from Argentina, $\mathrm{Al}$ falfa enamovirus-1 [6] (AEV-1) that was detected in alfalfa plants showing dwarfism symptoms; an AEV isolate from Sudan, designated AEV-2 [7]; Alfalfa virus S, a new species of the family Alphaflexiviridae discovered in alfalfa samples exhibiting chlorosis and stunting [8]; alfalfa virus F, a new member of the genus Marafivirus [9]; and Alfalfa leaf curl virus found in plants displaying leaf curling symptoms [10]. Alfalfa appears to be widely infected with seed-transmitted partitiviruses and the 
biological significance of these in alfalfa is currently unknown and requires further investigation [11, 12].

Publicly available transcriptomic datasets have became a valuable tool for the discovery of new pathogens, particularly viral sequences [11, 13-16]. The retrieval of complete or nearly complete viral genomes from transcriptomic data improves our knowledge of the diversity and host range of these pathogens and provides essential tools for their diagnostics and characterization. In this study, we performed a systematic survey of alfalfa transcriptomic datasets publicly available at NCBI. The survey indicated that approximately $90 \%$ of Medicago sativa samples employed in the generation of the deposited datasets contained viruses. Several emerging viruses were identified that had not been reported to infect alfalfa prior to this study or had not been experimentally confirmed in the plant.

\section{Methods}

Viral sequences were identified in alfalfa datasets retrieved from the NCBI Sequence Read Archive (https:// www.ncbi.nlm.nih.gov/sra). The raw sequencing reads were first mapped to the reference genomes of Medicago sativa (http://www.medicagohapmap.org/downloads/ cadl) and Medicago truncatula (http://www.medicagogenome.org/) with the Bowtie2 software described below. Those reads that did not map to the reference genomes were assembled into contigs using SPAdes (v.3.12.0) and searched (BLASTn with default setting and the following parameters: 20 threads, two alignments and two descriptions) against other plant genomes (rice, apple, beet, arabidopsis and tomato) to check for possible cross-run contamination. Reads that did not map to any plant species were aligned to the NCBI viral genome database (https://www.ncbi.nlm.nih. gov/genome/viruses/). The alignments were performed using BBMap (v. 37.66), SeqMan Ngen (version 15.2.0, build number 130; mer size 17-21, minimum match percentage 85\%) and Bowtie 2 (v.2.3.4) with the following very sensitive settings: -D $20-\mathrm{R} 3-\mathrm{N} 0-\mathrm{L} 20$-i S, 1 , 0.50 and $-\mathrm{p} 20$ (threads). In general, the very sensitive settings increase the search effort of Bowtie2 (D, R) by increasing the cutoffs for which Bowtie would stop searching. The length of the seed substring (L) was shortened to 20 from 22 and the interval (i) between substrings was decreased to 0.50 . The reads that were mapped to the reference viral sequences related to the viruses of interest and to the assembled viral contigs from the datasets, were sequestered and assembled de novo using SPAdes (v.3.12.0) [17] with the following parameters: kmers $21,31,41,51,81,91,95,-\mathrm{m} 800$-t 20 . Wet lab experiments to confirm the identified viral sequences were performed only with Medicago sativa amalgavirus 1 (MsAV1). Plant materials infected with other viruses were not available for experimental confirmation of the transcriptomic data. For the MsAV1 experiments, the same RNA samples employed in Nemchinov et al. [18] were used. Total RNA was extracted as previously described [18]. The $5^{\prime}$ end and 3' end of MsAV1 were amplified using the SMARTer RACE 5'/3' Kit (Takara Bio USA, Inc., Madison. WI). The primers used for $5^{\prime} /$ 3' RACE and the specific RT-PCR detection of the virus are listed in the Additional file 1. Phylogenetic trees were constructed using MEGA 7 software [19] by applying maximum likelihood method (with 1000 replicates) based on the JTT matrix-based model. The protein sequences were aligned by Cluster $\mathrm{W}$ incorporated into MEGA7. The sequence pairwise identity analysis of MsAV1 RdRp sequences was performed using the MAFFT program of the Sequence Demarcation Tool (http://web.cbio.uct.ac.za/ brejnev/). The SIAS tool that calculates pairwise sequence identity and similarity from multiple sequence alignments (http://imed.med.ucm.es/ Tools/sias.html) was used to predict amino acid identities between the Pro-Pol regions of cnidium vein yellowing virus (CnVYV) and lychnis mottle virus (LycMoV).

\section{Results}

We performed a systematic survey of 655 alfalfa transcriptomic datasets publicly available from the NCBI. The survey indicated that approximately $90 \%$ of Medicago sativa samples employed in the generation of the deposited datasets contained viruses. Twenty three different viruses were found in these transcriptomes, including several viruses not previously reported in alfalfa. The heterogeneity of the viral contigs identified in this study is summarized in Fig. 1 (see Additional file 2 for details). Essentially complete viral genomes were assembled for three emerging alfalfa viruses that have not been identified in alfalfa prior to this study (CnVYV, LycMoV and Cactus virus $X$ ) and for one virus that had not been experimentally confirmed in the plant (MsAV1).

\section{Medicago sativa amalgavirus 1 (MsAV1)}

The Medicago sativa amalgavirus 1 sequence was originally reported by Wang and Zhang in 2013 under the GenBank accession GAFF01077243.1 and later dubbed MsAV1 by Nibert et al. [20]. Prior to this study, the virus was not found in the US and its sequence had not been experimentally confirmed.

The RNA-seq data in which the virus reads were discovered, derived from the publicly available datasets SRR6050922 to SRR6050957 generated in our laboratory from the US alfalfa cultivars Maverick and ZG9830 [18]. The identified raw viral reads were mapped to the reference genome of MsAV1 (GAFF01077243.1; NC_ 040591.1) [20, 21] by Bowtie2 [22]. Each assembled 


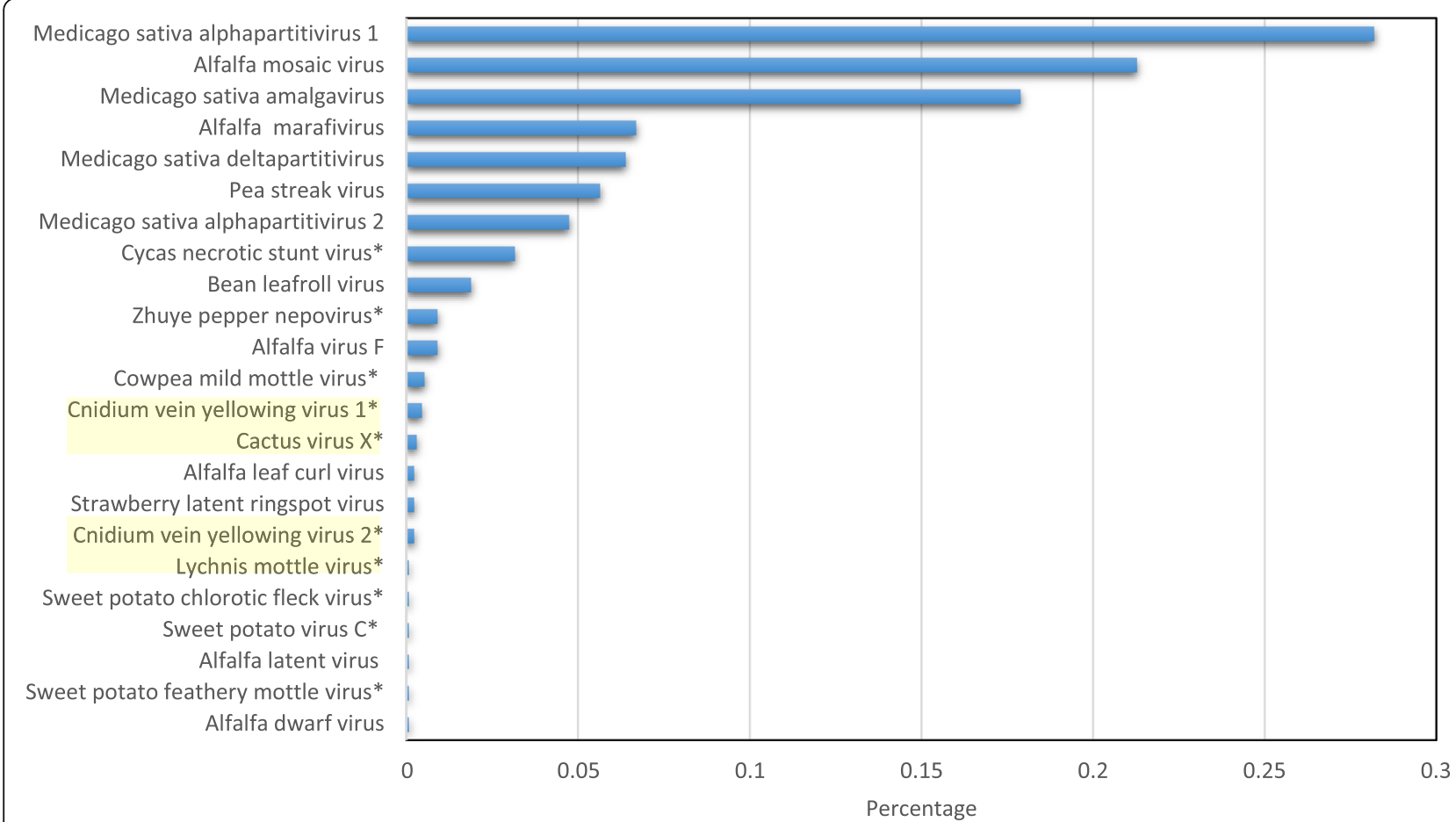

Fig. 1 Virome heterogeneity found in 655 publicly available alfalfa transcriptomic datasets. ${ }^{*}$ indicates viruses not previously reported in alfalfa. Highlighted are viruses that were characterized in this study

transcriptome was individually searched for amalgavirus sequences using the BLAST tool [23]. The numbers of amalgavirus reads found in the datasets are listed in Fig. 2. Among the 36 screened alfalfa datasets described in details in [18], half included MsAV1 reads (Fig. 2). In addition, the assembled contigs were searched with BLASTX against a database of the RNA-dependent RNA polymerase (RdRp) motif sequences of known RNA viruses as described in Kim et al. [11] (2018). The resulting viral contigs were assembled into a complete viral

\begin{tabular}{|c|c|c|c|c|}
\hline Sample No. & SRR No. & Virus reads No. & $\begin{array}{c}\text { RNA seq total } \\
\text { reads }\end{array}$ & Cultivar \\
\hline LN1 & SRR6050954 & 91 & 63964539 & Maverick \\
\hline LN2 & SRR6050955 & 3459 & 81355146 & Maverick \\
\hline LN6 & SRR6050948 & 2682 & 95342248 & Maverick \\
\hline LN7 & SRR6050947 & 2423 & 100911954 & Maverick \\
\hline LN8 & SRR6050946 & 120 & 78293205 & Maverick \\
\hline LN9 & SRR6050945 & 1827 & 53298114 & Maverick \\
\hline LN11 & SRR6050931 & 3754 & 98777042 & Maverick \\
\hline LN12 & SRR6050930 & 1609 & 42671096 & Maverick \\
\hline LN14 & SRR6050928 & 2177 & 90852091 & Maverick \\
\hline LN15 & SRR6050933 & 147 & 69368846 & Maverick \\
\hline LN18 & SRR6050941 & 2014 & 65342463 & Maverick \\
\hline LN19 & SRR6050942 & 4712 & 100110710 & ZG9830 \\
\hline LN23 & SRR6050937 & 3 & 56904390 & ZG9830 \\
\hline LN24 & SRR6050925 & 1319 & 64137356 & ZG9830 \\
\hline LN32 & SRR6050935 & 1312 & 137406222 & ZG9830 \\
\hline LN33 & SRR6050932 & 65 & 95845839 & ZG9830 \\
\hline LN34 & SRR6050940 & 465 & 109819523 & ZG9830 \\
\hline LN36 & SRR6050949 & 34 & 88971414 & ZG9830 \\
\hline
\end{tabular}

Fig. 2 Medicago sativa amalgavirus 1 (MsAV1) reads found in alfalfa datasets 
genome. The $5^{\prime} / 3^{\prime}$-terminal sequences of the viral genome were determined experimentally by RACE (Fig. 3 a). The MsAV1 genome consisted of a single molecule of dsRNA that was $3423 \mathrm{nt}$ in length and contained two putative overlapping open reading frames (ORF): ORF1, which encoded coat protein (CP) and ORF2, which encoded RNA-dependent RNA polymerase (RdRP) (Fig. 4). The 5' UTR was 129 nt-long and a the 3' UTR was $116 \mathrm{nt}$-long. The viral ORF1 (CP) was $1184 \mathrm{nt}$-long and a ORF2 (RdRP) was $3177 \mathrm{nt}$-long. The putative +1 ribosomal frameshifting motif of MsAV1 was UUUCGCA and was found at the nucleotide positions 985-991 (Fig. 4) suggesting that the viral RdRp encoded by ORF2 is expressed as a fusion protein via a ribosomal frameshift mechanism [20]. RT-PCR with virus-specific primers designed based on the in silico-generated sequence confirmed the virus identity (Fig. $3 \mathrm{~b}$ ).

Pairwise comparison of the MsAV1 RdRP sequences with other sequences in the family Amalgaviridae showed that RdRp is highly diverse (Fig. 5). Phylogenetic results based on the alignment of MsAV1 RdRP with that of other amalgaviruses suggested that MsAV is more related to the cleome droserifolia amalgavirus 1 (YP009553342), unclassified member of the family Amalgaviridae [20] (Fig. 6). The U.S. isolate of MsAV1 was $100 \%$ identical to the isolate GAFF01077243.1/ NC040591 from China $[20,21]$ at both the nucleotide and amino acid levels, indicating the same origin of the virus. This is the first experimental confirmation of MsAV1 infection in alfalfa in the United States, which is significant because amalgaviruses are known to be vertically transmitted through seeds.

\section{Cnidium vein yellowing virus}

Cnidium vein yellowing virus (CnVYV) is a bipartite, linear positive-sense ssRNA virus, a tentative member of the family Secoviridae, order Picornavirales [24]. Two isolates of the virus, CnVYV-1 and CnVYV-2, were found to infect cnidium plants (Cnidium officinale) in Korea [24]. To the best of our knowledge, no other hosts for CnVYV have been reported. Currently, the virus is not listed by ICTV as either an established or an unassigned species $[25,26]$.

For this study, the datasets were retrieved from NCBI Sequence Read Archive (SRA) and the contigs were individually assembled as described in the Materials and Methods. A total of 603,368 out of $108,082,074$ (0.55\%) raw Illumina pair-ended reads were found in accessions SRR2089795 and SRR2089796 of the BioProject PRJNA289195 [27] that mapped to the genome of CnVYV (GenBank accessions numbers: KR011028, KR011029, KR011030 and KR011031). When both datasets (SRR2089795 and SRR2089796) were checked for possible cross-run contamination, no plant species other than Medicago spp. were detected: $96 \%$ of the contigs had BLAST hits that corresponded to either M. sativa or M. truncatula.

The virus reads from both accessions SRR2089795 $(284,134$ reads out of $51,471,740)$ and SRR2089796 (342, 594 reads out of a total of 56,610,334 reads) were assembled into coding-complete bipartite viral genomes, consisting of RNA1 and RNA 2 segments from two different strains of CnVYV-related virus. The virus strains were provisionally designated CnVYV-A1 and CnVYV-A2.

The genome of CnVYV-A1 assembled from the accession SRR2089795, had two RNA molecules of 6983 and 3588 nucleotides in length, respectively, excluding the poly (A) tails (see Additional file 3). At the nucleotide level, the RNA 1 segment of the CnVYV-A1 had $75.6 \%$ identity (coverage $71 \%, \mathrm{E}$ value $=0.0$;) to RNA1 from the CnVYV-1 isolate (KR011028.1), which was the top BLAST hit for the CnVYV-A1.

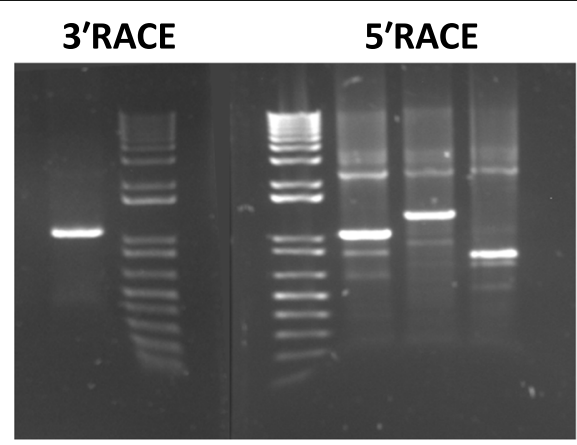

A

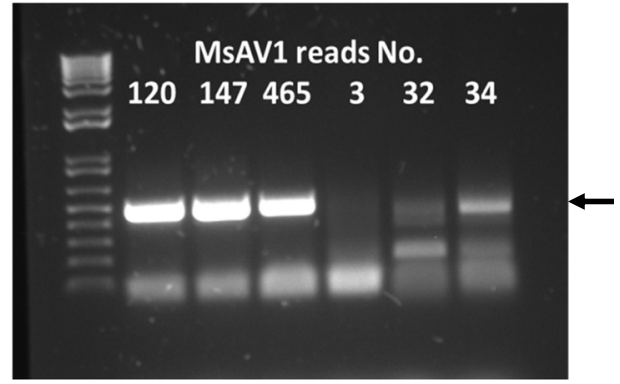

B

Fig. 3 a, 3' RACE and 5' RACE PCR products generated from the MsAV1-infected tissues. Lane 1: 3' RACE product amplified with a virus-specific primer GSP 2 and a 3'-CDS primer from the SMARTer RACE kit. Lanes 3,4, and 5: 5'RACE products amplified with different virus-specific primers (GSP1-1, GSP1-2 and GSP1-3, respectively) and a 5'-CDS primer from the SMARTer RACE kit; M: 1 kb Plus DNA Ladder (ThermoFisher Scientific, MA, USA). b, RT-PCR results obtained using primers MSAV1 F1 and MsAV1 R1 from selected alfalfa samples that had a low number of the MsAV1 reads. The arrow indicates $R T-P C R$ amplicons of the expected size (496 bp) 


\section{MsAV1 dsRNA}

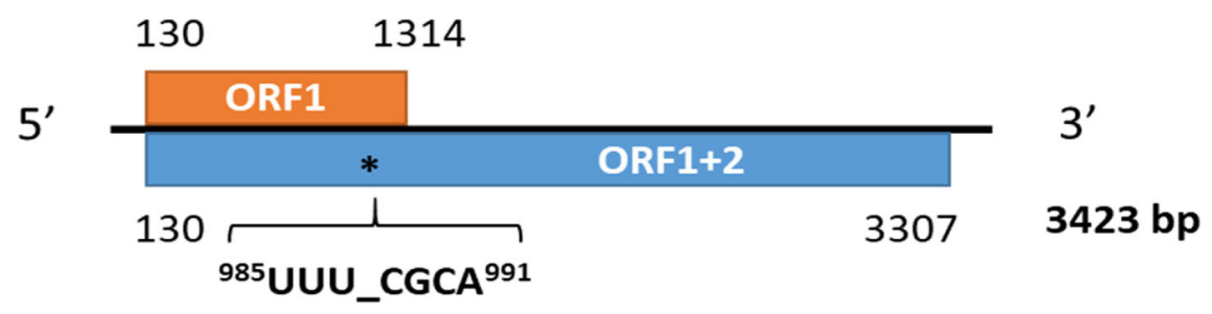

Fig. 4 Schematic representation of the genome organization of Medicago sativa amalgavirus1 and the putative +1 programmed ribosomal frameshifting motif in MsAV1

Rhododendron_virus_A_YP003868436 Camellia_oleifera_amalgavirus_1_YP009551564 Gevuina_avellana_amalgavirus_1_DAB41733 Cleome_droserifolia_amalgavirus_1_YP009553342 MsAV1

Secale_cereale_amalgavirus_1_DAB41729 Anthoxanthum_odoratum_amalgavirus_2_DAB41668 Spinach_amalgavirus_1_ARO72612

Salicornia_europaea_amalgavirus_1_DAB41669 Erigeron_breviscapus_amalgavirus_1_YP009552087 Rubber_dandelion_latent_virus_2_AWH55632 Erigeron_breviscapus_amalgavirus_2_YP009552089 Allum_cepa_amalgavirus_1_YP009447919 Allium_cepa_amalgavirus_2_YP009447921 Phalaenopsis_equestris_amalgavirus_1_YP009552083 Cucumis_melo_amalgavirus_1_QBC66123 Festuca_pratensis_amalgavirus_1_YP009552799 Anthoxanthum_odoratum_amalgavirus_1_YP009551562 Southern_tomato_virus_YP002321509 Capsicum_annuum_amalgavirus_1_YP009552797 Pinus_patula_amalgavirus_1_DAB41737 Festuca_pratensis_amalgavirus_2_YP009553344 Festuca_pratensis_amalgavirus_3_DAB41731 Lolium_perenne_amalgavirus_1_DAB41735 Blueberry_latent_virus_YP003934623 Zostera_marina_amalgavirus_1_YP009362302 Zostera_marina_amalgavirus_2_YP009362304
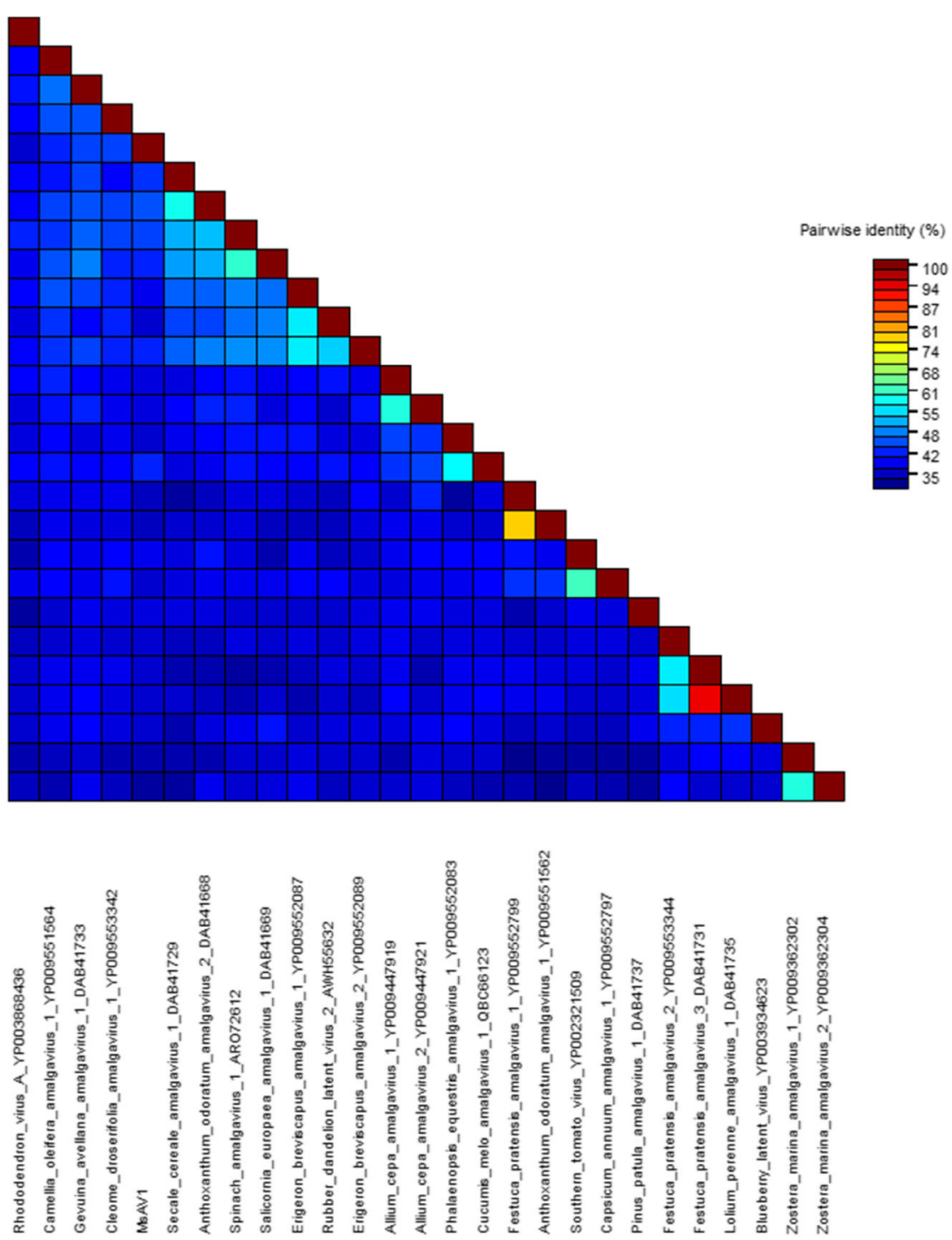

Fig. 5 The pairwise identity plot of RdRps in the family Amalgaviridae. The alignment was performed using MAFFT software and visualized by the Sequence Demarcation Tool (http://web.cbio.uct.ac.za/ brejnev/) 


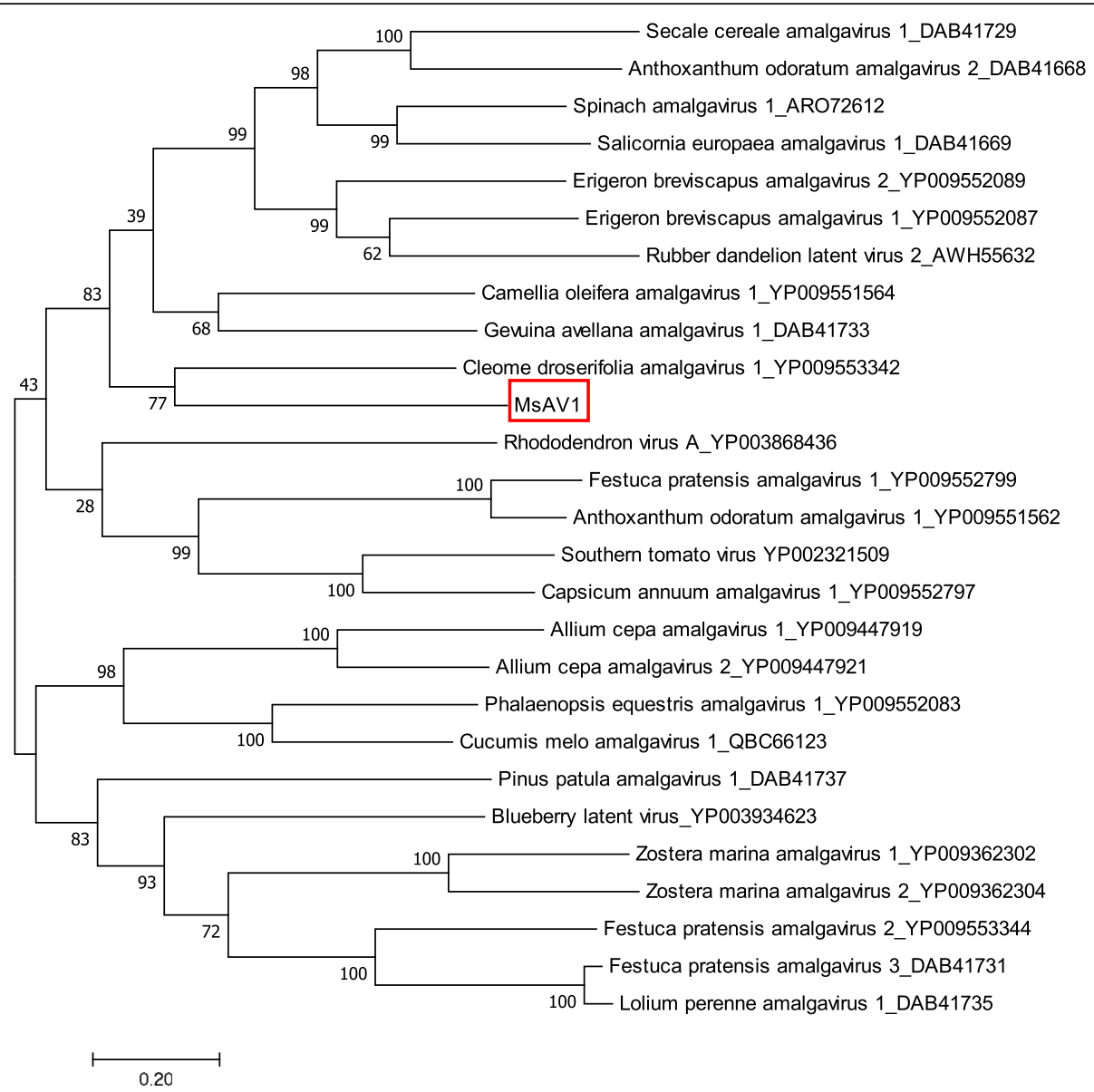

Fig. 6 Phylogenetic relationships between MsAV1 and other members of the family Amalgaviridae, based on the alignment of the amino acid sequences of their RdRPs. The trees were constructed using the Maximum Likelihood method of MEGA 7 [19] with 1000 bootstrap replicates

The RNA1 segment of the CnVYV-A1 strain contained a single ORF encoding for a putative polyprotein of the 2201 amino acids long (P1). The P1 had 79.6\% amino acid identity to the polyprotein 1 of CnVYV-1 (AKN59243.1). Other top BLAST hits included polyproteins from the CnVYV-2 isolate (AKN59245.1) $(79.07 \%$ identity, coverage $100 \%$, E value $=0.0)$, lychnis mottle virus $(\mathrm{LycMoV})$, (coverage 100\%; E-value $=0.0$; identity 77.5\%; accession AKN59247.1) and strawberry latent ringspot virus (SLRSV), (coverage 100\%; $\mathrm{E}$-value $=0.0$; identity 77.6\%; accession YP_227367.1). Thus, BLAST searches based on the amino acid alignments of P1 indicated the close relationship of the CnCVYV-A1 not only to the reference CnCVYV strains (1 and 2), but also to LycMoV and SLRSV. LycMoV is a tentative member of the family Secoviridae and SLRSV is an unassigned species in the same family [25].

The RNA 2 segment of the CnVYV-A1 sequence retrieved from the accession SRR2089795 was 77.03\% identical to the RNA 2 from LycMoV isolate Andong [28] (coverage 89\%, E-value $=0.0$; KR011033.1) at the nucleotide level. CnVYV-2 was the second closest match with a percent identity of $77 \%$ (coverage $58 \%$, E value = 0.0; KR011031.1). RNA2 encoded a polyprotein that was 994 aa-long (P2) which, based on the BLASTP search, was most closely related to LycMoV (coverage 100\%; Evalue $=0.0$; identity 92.45\%; accession AKN59248.1) . Other top hits included SLRSV (coverage 100\%; E-value $=0.0$; identity 70.2\%; YP_227368.1) and CnVYV2 (coverage 80\%; E-value $=0.0$; identity $88.5 \%$; AKN59246.1).

The numbers were slightly different for the CnVYVA2 strain, which was retrieved from the accession SRR2089796 (see Additional file 3). The RNA1 segment of the CnVYV-A2 strain was $6892 \mathrm{nt}$ long, excluding the poly(A) tail. At the nucleotide level, it was $71 \%$ identical to the complete RNA 1 of the CnVYV-1 Yeongyang isolate (coverage 71\%; E-value $=0.0$; KR011028.1). RNA1 of the CnVYV-A2 strain encoded a polyprotein of 2201 aalong that was $78.9 \%$ identical to that of the CnVYV-1 Yeongyang isolate (coverage 100\%; $\mathrm{E}$-value $=0.0$; AKN59243.1). Other top BLAST hits included LycMoV (coverage $100 \%$; E-value $=0.0$; identity $77.53 \%$; accession AKN59247.1) and CnVYV-2 (coverage 100\%; E-value = 
0.0; identity 78.89\%; AKN59245.1). The nucleotide and polyprotein sequences of the RNA 1 segments of the CnVYV-A1 and CnVYV-A2 strains were 90.6 and $96.6 \%$ identical, respectively.

The RNA 2 of the CnVYV-A2 strain, which was retrieved from the accession SRR2089796 was 3611 nucleotides long, excluding the poly(A) tail. It was $77.29 \%$ identical to the RNA 2 of the CnVYV-2 Yeongyang isolate (coverage 57\%, E-value =0.0; accession KR011031.1). Other BLAST hits (LycMoV and CnVYV 1) had a low coverage (1\%). The RNA 2 translated into a polyprotein of 994 aa-long, which, similar to the P2 in CnVYV-A1, showed a top BLAST hit for LycMoV (coverage 100\%; E-value $=0.0$; identity 90.54\%; accession BBE07891.1). Other closely related species were SLRSV (coverage $100 \%$; E-value $=0.0$; identity $70.01 \%$; YP_227368.1) and CnVYV 2 (coverage 80\%; E-value $=0.0$; identity $87.73 \%$; AKN59246.1). The RNA 2 segments of the CnVYV-A1 and A2 strains were 90.7 and $97.9 \%$ identical at the nucleotide and amino acid levels, respectively.

A Pfam database search (version 32.0) [29] of the CnVYV-A strains identified two conserved domains in their P1: RNA helicase (PF00910; E-value $=2.1 \mathrm{e}-28$ ) and RdRp (PF00680; E-value $=1.5 \mathrm{e}-60$ ). The putative cleavage sites between these proteins were all of the serine/ glycine type $(\mathrm{S} / \mathrm{G})$, as was observed in the CVYV-1 and CVYV-2 isolates. InterPro scan (version 5.36-75.0) [30] predicted two CP domains typical for RNA 2 polyproteins (IPR029053) in P2 of the CnVYV-A. The SIAS tool [31] detected several conserved domains in P2 of the CnVYV-A strains that were characteristic of $\mathrm{CP}(\mathrm{s})$ signatures found in secoviruses. Based on these predictions, we anticipated that CnVYV-A strains share a similar genome organization with other closely related viruses of the family Secoviridae (Fig. 7).
We thus concluded that CnVYV-A1 and CnVYV-A2 represent isolates of the same virus strain adapted to alfalfa, for which we propose the name CnVYV-A (alfalfa).

\section{Lychnis mottle virus}

Lychnis mottle virus (LycMoV) is a tentative member of the family Secoviridae that was first reported in Lychnis cognata, a flowering plant in the family Caryophyllaceae [28]. In 2017, the virus was also isolated from the leaves of Vincetoxicum acuminatum in Japan and the complete nucleotide sequence of LycMoV-J was obtained [32].

A total of 400,676 out of 56,610,334 (0.7\%) raw Illumina paired-end reads were found in the accession SRR2089796 [27] that mapped to the genome of the LycMoV isolate Andong (GenBank accessions numbers KR011032 and KR011033). The genome of the alfalfa isolate of LycMoV (LycMoV-A) that was assembled from the accession SRR2089796, consisted of two segments of 6972 and 3584 nucleotides in length (corresponding to RNA1 and RNA 2, respectively), excluding the poly (A) tails and appeared to be coding-complete (see Additional file 3).

BLAST searches showed that the RNA1 segment of LycMoV-A had $74 \%$ identity to that of the LycMoV Andong isolate (coverage $79 \%$; $\quad E$-value $=0.0$; KR011032.1). SLRSV was the second hit with a low query coverage (22\%). However, when the RNA 1 was translated into a putative polyprotein 2201 aa in length and analyzed by BLAST, the top hit was CnVYV-1 (score 3631; coverage 100\%; E-value $=0.0$; identity78.70\%; accession AKN59243.1). LycMoV was the second highest hit (score 3615; coverage 100\%; Evalue $=0.0$; identity 79.07\%; $\mathrm{BBE} 07890.1$ ). A translation of the nucleotide sequence presumably corresponding to LycMoV, into an amino acid sequence resembling that

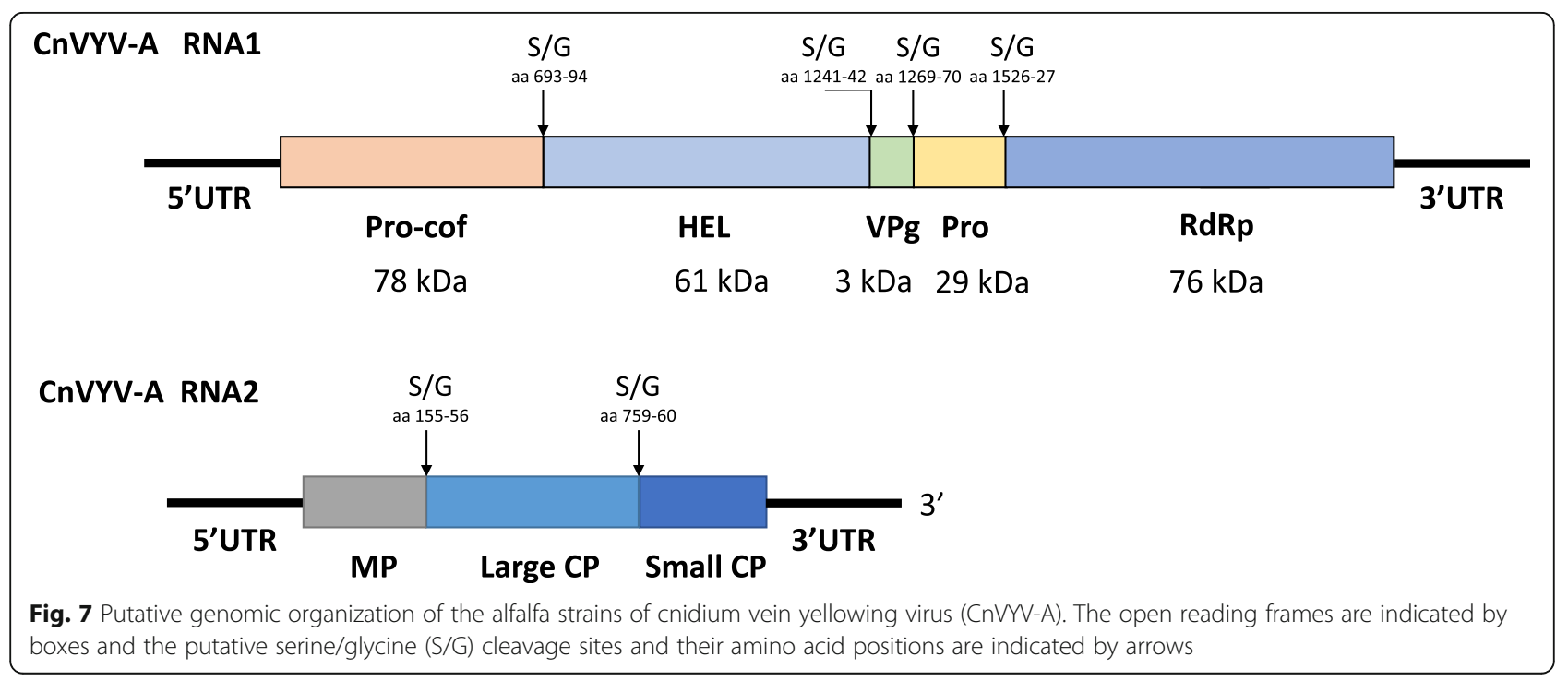


of the CnVYV suggested that CnVYV and LycMoV represent the same viral species.

The RNA 2 segment of LycMoV-A was 77.3\% identical to that of the LycMoV Andong isolate at the nucleotide level (coverage 89\%; E-value $=0.0$; KR011033.1). At the amino acid level, P2 had $90.9 \%$ percent identity with that of the LycMoV-J isolate (coverage 100\%; E-value $=0.0$; accession BBE07891.1). The Andong isolate of LycMoV (92.3\% identity; AKN59248.1) was the second highest hit and SLRSV (70.1\% identity; YP_227368.1) was the third highest.

\section{Relationship between the CnVYV strains and LycMoV}

To determine whether LycMoV and CnVYV are strains of the same virus species or correspond to the distinct species as was suggested by Yoo et al. [24, 28] for the Yeongyang isolates of CnVYV from Cnidium officinale and the Andong isolate of LycMoV from Lychnis cognata, we compared the amino acid identities between their Pro-Pol and CP regions, which are currently used as species demarcation criteria by the ICTV [25]. The SIAS tool [31] predicted the following amino acid identities between the Pro-Pol regions of these viruses (Fig. 8). Although the identities were slightly different from the ones generated by BLASTP due to the different formula used for the calculations, all the conserved ProPol values were higher than $80 \%$ (the ICTV criteria for species demarcation are less than $80 \%$ identity) and all the CP values, except that for SLRSV, were higher than $75 \%$ (ICTV criteria for species demarcation are below
75\% identity). We speculate that CnVYV-A, together with all the other viruses in this study group (CnVYV-1, CnVYV-2, LycMoV-A, LycMoV and LycMoV-J) and with the exception of SLRSV due to the low identity of its $\mathrm{CP}$ region, constitute individual strains of the same viral species isolated from different hosts, for which a collective name reflecting its biological characteristics and taxonomic position is required. We suggest the following temporary name for the species: cnidium vein yellowing-like virus (CnVYLV).

Phylogenetic analyses based on the alignments of the Pro-Pol region between the protease CG motif and the RdRp GDD motif (CG/GDD) in RNA1 and the predicted $\mathrm{CP}$ region in the RNA 2 segment supported this conclusion: CnVYV-A1 and CnVYV-A2 were grouped together with the reported strains of the CnVYV and LycMoV (Fig. 9) and the cluster branched out toward a more diverse strawberry latent ringspot virus.

\section{Cactus virus $\mathbf{X}$}

Cactus virus $X(\mathrm{CVX})$ is a member of the family Alphaflexiviridae, genus Potexvirus. It infects many species in the plant family Cactaceae worldwide [33]. Prior to this report, it had not been identified in alfalfa. The NCBI reference sequence for CVX was reported under two identical accessions NC_002815.2 and AF308158 [34].

In this work, we have detected 2028 paired-end CVXrelated reads in the publicly available alfalfa transcriptomic dataset SRR7751381 from the NCBI BioProject PRJNA487676 [35]. The raw reads were assembled de

\begin{tabular}{|c|c|c|c|c|c|c|c|c|}
\hline CnVYV-Al & $100 \%$ & & & & & & & \\
\hline CnVYV-A2 & $98.23 \%$ & $100 \%$ & & & & & & \\
\hline CnVYV-1_AKN59243 & $91.15 \%$ & $90.7 \%$ & $100 \%$ & & & & & \\
\hline CnVYV-2_AKN59245 & $91.15 \%$ & $91.59 \%$ & $94.69 \%$ & $100 \%$ & & & & \\
\hline LycMoV-A & $98.45 \%$ & $98.67 \%$ & $90.7 \%$ & $91.15 \%$ & $100 \%$ & & & \\
\hline LycMoV_AKN59247 & $92.03 \%$ & $92.92 \%$ & $94.91 \%$ & $97.34 \%$ & $92.47 \%$ & $100 \%$ & & \\
\hline LycMoV-J_LC382243 & $91.15 \%$ & $91.59 \%$ & $95.57 \%$ & $94.46 \%$ & $91.15 \%$ & $95.13 \%$ & $100 \%$ & \\
\hline \multirow[t]{2}{*}{ SLRSV_YP227367 } & $91.15 \%$ & $91.15 \%$ & $90.7 \%$ & $92.03 \%$ & $90.92 \%$ & $92.03 \%$ & $91.37 \%$ & $100 \%$ \\
\hline & CnVYV-Al & CnVYV-A2 & CnVYV-1_AKN59243 & CnVYV-2_AKN59245 & LycMoV-A & LycMoV_AKN59247 & LycMoV-J_LC382243 & SLRSV_YP227367 \\
\hline & & & & $A$ & & & & \\
\hline CnVYV-Al & $100 \%$ & & & & & & & \\
\hline CvVYV-A2 & $96.94 \%$ & $100 \%$ & & & & & & \\
\hline CnVYV-1_AKN59244 & $89.31 \%$ & $89.05 \%$ & $100 \%$ & & & & & \\
\hline CnVYV-2_AKN59246 & $92.36 \%$ & $91.09 \%$ & $91.09 \%$ & $100 \%$ & & & & \\
\hline LycMoV-A & $98.47 \%$ & $98.47 \%$ & $90.07 \%$ & $92.62 \%$ & $100 \%$ & & & \\
\hline LycMoV_AKN59248 & $92.36 \%$ & $91.6 \%$ & $92.62 \%$ & $93.63 \%$ & $92.62 \%$ & $100 \%$ & & \\
\hline LycMoV-J_BBE07891 & $89.82 \%$ & $89.56 \%$ & $95.92 \%$ & $92.62 \%$ & $90.58 \%$ & $94.4 \%$ & $100 \%$ & \\
\hline \multirow[t]{3}{*}{ SLRSV_YP227375 } & $6.7 \%$ & $7.21 \%$ & $7.21 \%$ & $7.73 \%$ & $6.95 \%$ & $7.47 \%$ & $7.47 \%$ & $100 \%$ \\
\hline & CnVYV-Al & CvVYV-A2 & CnVYV-1_AKN59244 & CnVYV-2_AKN59246 & LycMoV-A & LycMoV_AKN59248 & LycMoV-J_BBE07891 & SLRSV_YP227375 \\
\hline & & & & B & & & & \\
\hline
\end{tabular}




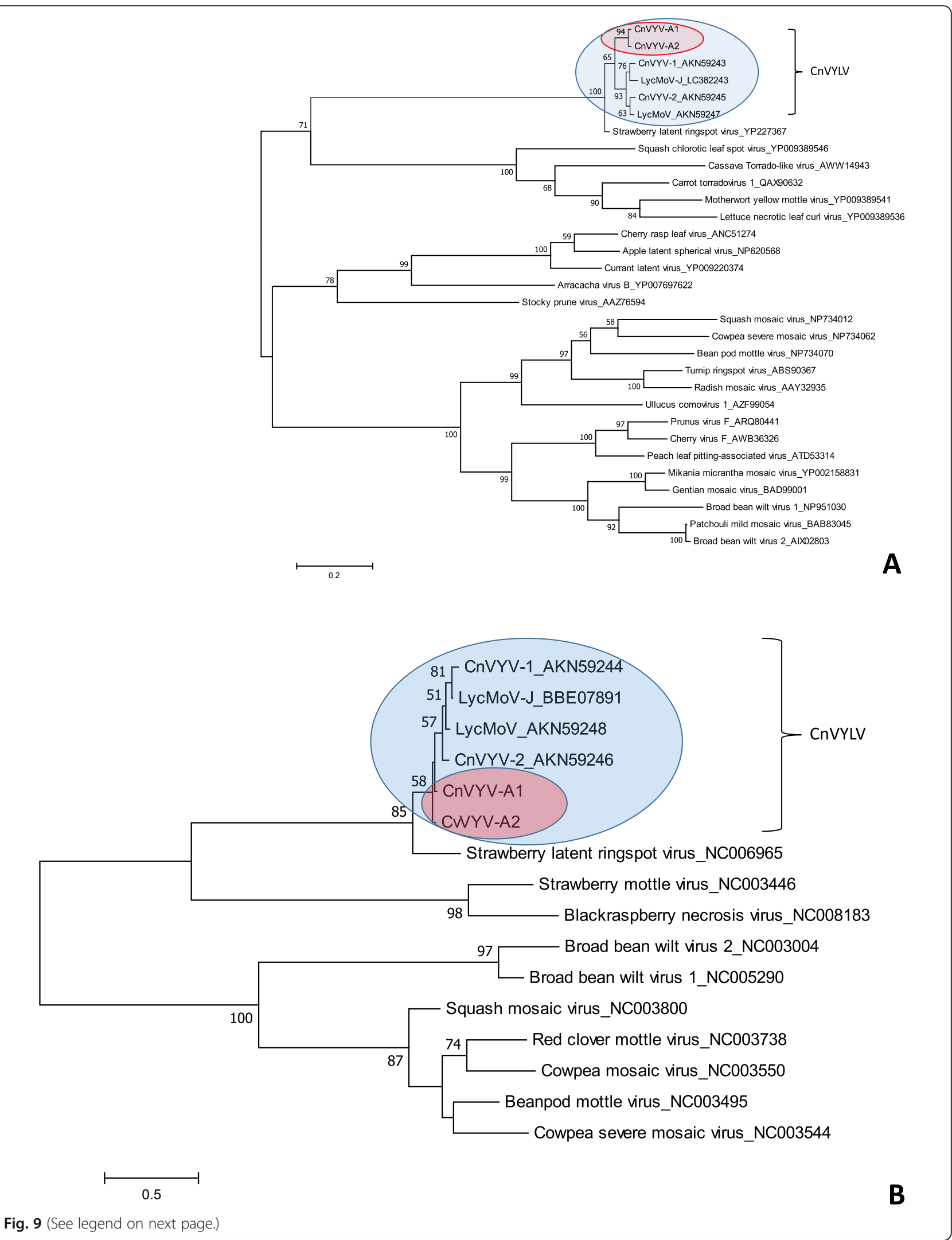


(See figure on previous page.)

Fig. 9 Phylogenetic analyses based on the amino acid alignments of the predicted Pro-Pol region of RNA1 $\mathbf{a}$ and the CP region of RNA 2 b of the CnVV-A strains and other members of the family Secoviridae. The trees were generated using the Maximum likelihood algorithm of MEGA7 [19] with 1000 bootstrap replicates. Blue oval indicates different viral strains that belong to the proposed species tentatively named cnidium vein yellowing-like virus (CnVYLV). Red oval indicates viral strains found in alfalfa

novo into a coding-complete virus genome consisting of a single molecule of linear ssRNA 6603 nucleotides in length, excluding the poly(A) tail (see Additional file 3). At the nucleotide level, the alfalfa isolate of CVX (CVX-A) was 97\% identical to the CVX reference sequence (coverage 100\%; $\mathrm{E}$-value $=0.0$; AF308158.2). Other BLAST hits included different CVX isolates with lower alignment scores.

The 5'UTR of the CVX-A consisted of 74 nucleotides preceding the initiation codon of ORF1 and began with the sequence CCAACACCAA, which is most likely missing a few nucleotides $(\sim 5 \mathrm{nt})$ at the very $5^{\prime}$ terminus. The 3'UTR consisted of 99 nucleotides downstream of the termination codon of the ORF5 (pos. 6502-6504) and appeared nearly complete, missing at most one or two nucleotides. Viral RNA translated into five putative ORFs encoding for RdRp (ORF1, 75-4703 nt); triple gene block protein 1 (TGBp1; ORF2, $25 \mathrm{kDa}$; 4703-5392 nt); partially overlapping with the ORF2 triple gene block protein 2 (TGBp2; ORF3; 5355-5687 nt; 11.9 kDa); triple gene block protein 3 overlapping with the ORF 3 (TGBp3; ORF4; $5617-5811 \mathrm{nt}$; $6.7 \mathrm{kDa}$ ); and a putative coat protein separated from the ORF 4 by a 15 nt-long intergenic region (ORF5; 5827-6504; 24.3 kDa).

We predicted that the genome organization of the CVX-A largely resembles that of the CVX [33]. BLASTP analysis of the aa identities of the putative CVX-A proteins with the corresponding proteins of the reference genome suggested their close relationship (Table 1). Phylogenetic analysis performed with the aa sequences of CVX-A RdRp and CP placed CVX-A in the same subcluster as CVX. We concluded that CVX-A represents a strain of CVX, adapted to alfalfa (Fig. 10).

\section{Discussion}

Publicly available transcriptomic datasets are becoming an increasingly valuable tool for the discovery of new pathogens, particularly viral sequences. In this study, we performed a systematic survey of 655 alfalfa transcriptomic datasets publicly available at the NCBI. Twenty three different viruses were found in the analyzed transcriptomes, including several emerging viral pathogens not previously reported in $M$. sativa prior to this study. Among them were two strains of cnidium vein yellowing virus, lychnis mottle virus and Cactus virus $X$, for which coding-complete genomic sequences were obtained by a de novo assembly. Based on the amino acid identities of the conserved Pro-Pol region, the CP sequences and the phylogenetic analyses, we suggested that CnVYV-A1, CnVYV-A2, CnVYV-1, CnVYV-2, LycMoV-A, LycMoV, and LycMoV-J represent different strains of the same viral species, tentatively called cnidium vein yellowing-like virus (CnVYLV). We have also presented the first in silico and experimental identification of Medicago sativa amalgavirus 1 in US alfalfa germplasm, which is significant because amalgaviruses are known to be vertically transmitted through seeds. Although amalgaviruses are not considered pathogenic, they could be of economic importance due to their potential role in mixed infections [36] and ubiquitous presence in some cultivars, species or genera of plants [37].

The reported results improve our knowledge of the diversity and host range of viruses infecting alfalfa and provide essential tools for their diagnostics and characterization.

\section{Conclusions}

The systematic survey of alfalfa transcriptomic datasets publicly available at the NCBI indicated that approximately $90 \%$ of Medicago sativa samples employed in the generation of the deposited datasets contained viruses. Several emerging viruses were identified that had not been reported to infect alfalfa prior to this study or had not been experimentally confirmed in the plant. Coding-complete genomic sequences were obtained for cnidium vein yellowing virus (CnVYV), lychnis mottle virus (LycMoV) and Cactus virus $X$ (CVX), all of which have not been diagnosed in alfalfa until now. Further research is required to confirm the in silico identification of these viruses and to determine their symptomatology, geographic distribution and economic importance to the alfalfa industry.

Table 1 BLASTP alignment scores of the CVX-A and CVX

\begin{tabular}{llllll}
\hline Alignment scores & RdRp & TGBp1 & TGBp2 & TGBp3 & CP \\
\hline Percent identity & $98.2 \%$ & $100 \%$ & $98.18 \%$ & $98.44 \%$ & $4 \mathrm{e}-36$ \\
E-value & 0.0 & 0.0 & $1 \mathrm{e}-71$ & 100 & $7 \mathrm{e}-163$ \\
Query cover & $100 \%$ & $100 \%$ & $100 \%$ & $100 \%$ & 100 \\
Accession \# & BAU68240.1 & NP_148781.1 & AlW81551.1 & AMX81286.1 & BAO73885.1 \\
\hline
\end{tabular}




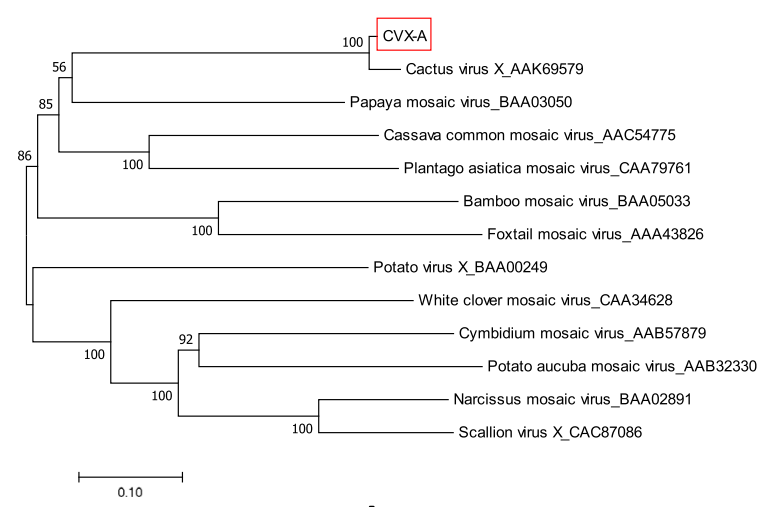

A

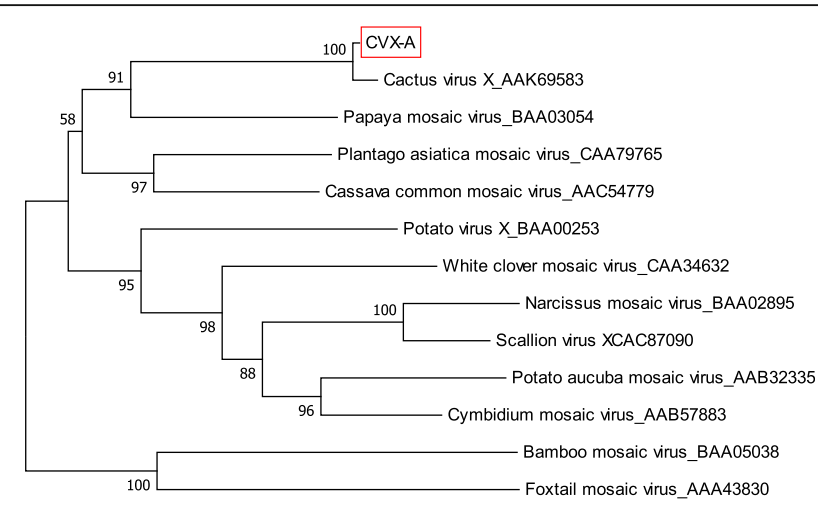

$\longmapsto .10$

Fig. 10 Phylogenetic analyses based on the amino acid alignments of the predicted RdRP $\mathbf{a}$ and the CP sequences $\mathbf{b}$ of CVX-A and other members of the family Potexviridae. The trees were generated using the Maximum likelihood algorithm of MEGA7 [19] with 1000 bootstrap replicates

\section{Supplementary information}

Supplementary information accompanies this paper at https://doi.org/10. 1186/s12985-019-1257-y.

Additional file 1: Primers used in the $5^{\prime} / 3^{\prime}$ RACE and RT-PCR reactions for amplification of the MsAV1-derived products.

Additional file 2: Overview of viral contigs identified in 655 alfalfa transcriptomic datasets.

Additional file 3: Nucleotide sequences of the alfalfa strains of cnidium vein yellowing virus (CnVYV-A), lychnis mottle virus (LycMoV-A) and Cactus virus $X(\mathrm{CVX}-\mathrm{A})$.

\section{Abbreviations}

CnVV: Cnidium vein yellowing virus; CVX: Cactus virus $X_{i}$ LycMoV: Lychnis mottle virus; MsAV1: Medicago sativa amalgavirus 1; RACE: Rapid amplification of CDNA ends; RT-PCR: Reverse transcription polymerase chain reaction

\section{Acknowledgements}

Not applicable.

\section{Authors' contributions}

LGN conceived the study. PJ and LGN performed the experiments. PJ and JS conducted bioinformatic investigations. LGN, PJ and JS analyzed the data. PJ wrote the first draft of the paper. LGN wrote the final version of the manuscript. All authors read and approved the manuscript before submission.

\section{Authors' information}

USDA/ARS, Beltsville Agricultural Research Center, Molecular Plant Pathology Laboratory, Beltsville, MD 20705. Corresponding author: Lev G. Nemchinov. lev.nemchinov@usda.gov

\section{Funding}

This research was supported by the United States Department of Agriculture, Agricultural Research Service, CRIS project 8042-21000-300-00D.

\section{Availability of data and materials}

All nucleotide sequences described in the manuscript are available in the Additional file 3. The sequences have also been deposited to the Third Party Annotation Section of the DDBJ/ENA/GenBank databases under the accession numbers TPA: BK011041-BK011047.
Ethics approval and consent to participate

Not applicable.

\section{Consent for publication}

Not applicable.

\section{Competing interests}

The authors declare that they have no competing interests.

Received: 12 September 2019 Accepted: 22 November 2019

Published online: 10 December 2019

\section{References}

1. Li X, Brummer EC. Applied genetics and genomics in alfalfa breeding. Agronomy. 2012;2:40-61.

2. National Alfalfa and Forage Alliance, http://alfalfa.org/newsletter/18032 7nafanews.htm. Accessed 27 Mar 2018.

3. Samac DA, Rhodes LH, Lamp WO. Compendium of Alfalfa Diseases and Pests. In: The American Phytopathological Society. 3rd ed. St. Paul: APS Press; 2015

4. Monteros MJ, Bouton JH. The future of alfalfa and forage crops in: proceedings. Reno: Western Alfalfa \& Forage Conference; 2009. p. 2-4.

5. Bejerman N, Nome C, Giolitti F, Kitajima E, de Breuil S, Fernandez JP, Basigalup D, Cornacchione M, Lenardon S. First report of a Rhabdovirus infecting alfalfa in Argentina. Plant Dis. 2011;95:771.

6. Bejerman N, Giolitti F, Trucco V, de Breuil S, Dietzgen RG, Lenardon S. Complete genome sequence of a new enamovirus from Argentina infecting alfalfa plants showing dwarfism symptoms. Arch Virol. 2016;161: 2029-32.

7. Nemchinov LG, Grinstead SC, Mollov DS. First report and complete genome sequence of alfalfa enamovirus from Sudan. Genome Announc. 2017;5: e00531-17.

8. Nemchinov LG, Grinstead SC, Mollov DS. Alfalfa virus S, a new species in the family Alphaflexiviridae. PLoS One. 2017;12:e0178222.

9. Nemchinov LG, François S, Roumagnac P, Ogliastro M, Hammond RW, Mollov DS, Filloux D. Characterization of alfalfa virus F, a new member of the genus Marafivirus. PLoS One. 2018;13:e203477.

10. Roumagnac P, Granier M, Bernardo P, Deshoux M, Ferdinand R, Galzi S, Fernandez E, Julian C, Abt I, Filloux D, Mesléard F, Varsani A, Blanc S, Martin $D P$, Peterschmitt M. Alfalfa leaf curl virus: an aphid-transmitted geminivirus. J Virol. 2015:89:9683-8.

11. Kim H, Park D, Hahn Y. Identification a novel RNA viruses in alfalfa (Medicago sativa): an Alphapartitivirus, Deltapartitivirus, and a Marafivirus. Gene. 2018;638:7-12. 
12. Nemchinov LG, Lee MN, Shao J. First Report of Alphapartitiviruses infecting alfalfa (Medicago sativa L.) in the United States. Microbiol Resour Announc. 2018;7:e01266-18.

13. Gilbert KB, Holcomb EE, Allscheid RL, Carrington JC. Discovery of new mycoviral genomes within publicly available fungal transcriptomic datasets. BioRxiv. 2019. https://doi.org/10.1101/510404.

14. Bekal S, Domier LL, Gonfa B, McCoppin NK, Lambert KN, Bhalerao K. A novel flavivirus in the soybean cyst nematode. J Gen Virol. 2014;95:1272-80.

15. Vieira P, Nemchinov LG. A novel species of RNA virus associated with root lesion nematode Pratylenchus penetrans. J Gen Virol. 2019;100:704-8.

16. Mushegian A, Shipunov A, Elena SF. Changes in the composition of the RNA virome mark evolutionary transitions in green plants. BMC Biol. 2016;14:68.

17. Bankevich A, Nurk S, Antipov D, Gurevich AA, Dvorkin M, Kulikov AS, Lesin VM, Nikolenko SI, Pham S, Prjibelski AD, Pyshkin AV, Sirotkin AV, Vyahhi N, Tesler G, Alekseyev MA, Pevzner PA. SPAdes: a new genome assembly algorithm and its applications to single-cell sequencing. J Comput Biol. 2012;19:455-77.

18. Nemchinov LG, Shao J, Lee MN, Postnikova OA, Samac DA. Resistant and susceptible responses in alfalfa (Medicago sativa) to bacterial stem blight caused by Pseudomonas syringae pv. syringae. PLoS One. 2017;12:e0189781.

19. Kumar S, Stecher G, Tamura K. MEGA7: molecular evolutionary genetics analysis version 7.0 for bigger datasets. Mol Biol Evol. 2016;33:1870-4.

20. Nibert $M L$, Pyle JD, Firth AE. A +1 ribosomal frameshifting motif prevalent among plant amalgaviruses. Virology. 2016;498:201-20.

21. Zhang S, Shi Y, Cheng N, Du H, Fan W, Wang C. 2015. De novo characterization of fall dormant and nondormant alfalfa (Medicago sativa L.) leaf transcriptome and identification of candidate genes related to fall dormancy. PLoS One. 2015;10:e0122170.

22. Langmead B, Salzberg SL. Fast gapped-read alignment with bowtie 2. Nat Methods. 2012;9:357-9

23. Altschul SF, Gish W, Miller W, Myers EW, Lipman DJ. Basic local alignment search tool. J Mol Biol. 1990;215:403-10.

24. Yoo RH. Zhao F, Lim S, Igori D, Kim SM, an TJ4, Lee SH, Moon JS. The complete genome sequences of two isolates of cnidium vein yellowing virus, a tentative new member of the family Secoviridae. Arch Virol. 2015; 160:2911-4.

25. Virus Taxonomy: The classification and nomenclature of viruses. The ICTV Report https://talk.ictvonline.org/ictv-reports/ictv_online_report. Accessed 27 Mar 2018

26. Thompson JR, Dasgupta I, Fuchs M, Iwanami T, Karasev AV, Petrzik K Sanfaçon H, Tzanetakis IE, van der Vlugt R, Wetzel T, Yoshikawa N, ICTV Report Consortium. ICTV Virus Taxonomy Profile: Secoviridae. J Gen Virol. 2017:98:529-31.

27. Song $L$, Jiang $L$, Chen $Y$, Shu $Y$, Bai $Y$, Guo C. Deep-sequencing transcriptome analysis of field-grown Medicago sativa L. crown buds acclimated to freezing stress. Funct Integr Genomics. 2016;16:495-511.

28. Yoo RH, Zhao F, Lim S, Igori D, Lee S-H, Moon JS. The complete nucleotide sequence and genome organization of lychnis mottle virus. Arch Virol. 2015; 160:2891-4.

29. El-Gebali S, Mistry J, Bateman A, Eddy SR, Luciani A, Potter SC, Qureshi M, Richardson LJ, Salazar GA, Smart A, Sonnhammer ELL, Hirsh L, Paladin L, Piovesan D, Tosatto SCE, Finn RD. The Pfam protein families database in 2019. Nucleic Acids Res. 2019;47:D427-D43.

30. Mitchell AL, Attwood TK, Babbitt PC, Blum M, Bork P, Bridge A, Brown SD, Chang HY, El-Gebali S, Fraser MI, Gough J, Haft DR, Huang H, Letunic I, Lopez R, Luciani A, Madeira F, Marchler-Bauer A, Mi H, Natale DA, Necci M, Nuka G, Orengo C, Pandurangan AP, Paysan-Lafosse T, Pesseat S, Potter SC, Qureshi MA, Rawlings ND, Redaschi N, Richardson L, Rivoire C, Salazar GA, Sangrador-Vegas A, CJA S, Sillitoe I, Sutton GG, Thanki N, Thomas PD, SCE T, Yong SY, Finn RD. InterPro in 2019: improving coverage, classification and access to protein sequence annotations. Nucleic Acids Res. 2019. https://doi. org/10.1093/nar/gky11002

31. SIAS, Sequence Identity And Similarity. Complutense University of Madrid. http://imed.med.ucm.es/Tools/sias.html. Accessed 5 Nov 2019.

32. Fujimoto Y, Nijo T, Hosoe N, Watanabe K, Maejima K, Yamaji Y, Namba S. Complete genome sequence of lychnis mottle virus isolated in Japan. Genome Announc. 2018;6(25):e00535-18.

33. Koenig R. In: Brunt AA, Crabtree K, Dallwitz MJ, Gibbs AJ, Watson L, Zurcher EJ, editors. Cactus $X$ potexvirus. Plant viruses online. Descriptions and lists from the VIDE Database; 1987. http://bio-mirror.im.ac.cn/mirrors/pvo/vide/ descr132.htm\#Range.
34. Liou MR, Chen YR, Liou RF. Complete nucleotide sequence and genome organization of a Cactus virus $X$ strain from Hylocereus undatus (Cactaceae). Arch Virol. 2004;149:1037-43.

35. Dong W, Liu X, Li D, Gao T, Song Y. Transcriptional profiling reveals that a MYB transcription factor MsMYB4 contributes to the salinity stress response of alfalfa. PLoS One. 2018;3:e0204033.

36. Martin RR, Zhou J, Tzanetakis IE. Blueberry latent virus: an amalgam of the Partitiviridae and Totiviridae. Virus Res. 2011;155:175-80.

37. Loebenstein G. Katis NI (eds). Control of plant virus diseases: vegetativelypropagated crops Adv Virus Res. 2015;91:2-319.

\section{Publisher's Note}

Springer Nature remains neutral with regard to jurisdictional claims in published maps and institutional affiliations.
Ready to submit your research? Choose BMC and benefit from:

- fast, convenient online submission

- thorough peer review by experienced researchers in your field

- rapid publication on acceptance

- support for research data, including large and complex data types

- gold Open Access which fosters wider collaboration and increased citations

- maximum visibility for your research: over $100 \mathrm{M}$ website views per year

At BMC, research is always in progress.

Learn more biomedcentral.com/submissions 